\title{
Numerical Geometry of Map and Model Assessment
}

\author{
Willy Wriggers ${ }^{1}$ and Jing $\mathrm{He}^{2}$ \\ ${ }^{1}$ Department of Mechanical \& Aerospace Engineering, Old Dominion University, Norfolk, VA, 23529 \\ ${ }^{2}$ Department of Computer Science, Old Dominion University, Norfolk, VA, 23529 \\ 1,2 co-corresponding authors: \{wwrigger, jhe\}@odu.edu
}

\section{Abstract}

We are describing best practices and assessment strategies for the atomic interpretation of cryo-Electron Microscopy (cryo-EM) maps. Multiscale numerical geometry strategies in the Situs package and in secondary structure detection software are currently evolving due to the recent increases in cryo-EM resolution. Criteria that aim to predict the accuracy of fitted atomic models at low (worse than $8 \AA$ ) and medium (4-8 $\AA$ ) resolution remain challenging. However, a high level of confidence in atomic models can be achieved by combining such criteria. The observed errors are due to map-model discrepancies and due to the effect of imperfect global docking strategies. Extending the earlier motion capture approach developed for flexible fitting, we use simulated fiducials (pseudoatoms) at varying levels of coarse graining to track the local drift of structural features. We compare three tracking approaches: naïve vector quantization, a smoothly deformable model, and a tessellation of the structure into rigid Voronoi cells which are fitted using a multi-fragment refinement approach. The lowest error is an upper bound for the (small) discrepancy between crystal structure and EM map due to different conditions in their structure determination. When internal features such as secondary structures are visible in medium-resolution EM maps, it is possible to extend the idea of point-based fiducials to more complex geometric representations such as helical axes, strands, and skeletons. We propose a quantitative strategies to assess map-model pairs when such secondary structure patterns are prominent.

\section{Introduction}

In the past decade, significant progress was made in 3D imaging of macromolecular assemblies via cryoElectron Microscopy (cryo-EM) and in the development of computational algorithms that interpret the resulting volumetric maps at atomic resolution. Modern CMOS detectors combined with movie frame stacking have eliminated much of the specimen drift that has limited cryo-EM imaging using earlier detectors. The new direct detection camera technology is a major breakthrough toward higher-resolution cryo-EM reconstruction (Jin et al., 2008). The new imaging techniques enable the production of many 3D density maps that, at the highest resolution end of the scale (better than $3.4 \AA$ ) allow atomic structures to be solved directly. De novo models from high resolution maps ( $4 \AA$ or better) can also be improved with crystallographic refinement techniques. 

flexible fitting packages (López-Blanco and Chacón, 2015). In the following we describe features of the Situs package, which was first released in 1998 (Wriggers, 2012). The Protein Data Bank (PDB) uses the "Situs colores program to identify transformation matrices that allow spatial overlap of maps deposited to EM Data Bank (EMDB) and models deposited to PDB so that they can be viewed together," and finds "that the unique algorithm in colores is able to identify optimal fit positions even in tricky cases, e.g., where only part of a structure is modelled." (Lawson, 2014). Among 1,014 EMDB entries with an associated atomic model in the PDB (Lawson, 2014), modelers filled the optional software form field in 204 unique cases. The top six programs are as follows: 1. UCSF Chimera [(Pettersen et al., 2004); 61 models], 2. MDFF [(Trabuco et al., 2008); 28 models], 3. Situs (26 models), 4. EMFit [(Rossmann et al., 2001); 24 models], 5. Coot [(Emsley et al., 2010); 12 models], and 6. Rosetta [(DiMaio et al., 2009); 11 models]. We note that the top three programs are complementary (Chimera is widely used for visualization, MDFF is used for molecular dynamics simulations of high-resolution maps, and Situs is traditionally used for rigid-body docking at medium to low resolution).

In this whitepaper, our aim is to focus on opportunities, challenges, and aspirations as they relate to software developed in our laboratories. Numerical geometry methods are described in more detail in the Figure captions. We proceed in order of increasing cryo-EM resolution as follows. In Results and Discussion, we summarize first best practices of model evaluation inspired by practical uses of our Situs rigid-body docking tools. We next consider practical cases where the drift of local features can be evaluated due to the existence of a known atomic structure. We conclude with an outlook on detection of secondary structure features in medium to high-resolution EM maps, which holds much promise for future validation strategies.

\section{Results and Discussion}

We begin with an overview of the existing state of the art in validation criteria for low to medium resolution fitted models, before advancing to new ideas for the basis of current and future research.

\section{Low Resolution: Existing Best Practices for Rigid-Body Fitting}


Even in the age of atomic resolution cryo-EM, the fitting of small fragments to low resolution maps can still be very valuable, as has been exemplified by the recent fitting of the newly solved structure of the so-called SPRY2 domain of ryanodine receptor (RyR1) to four low (worse than $10 \AA$ ) resolution maps (Lau and Van Petegem, 2014). The fragment was docked using colores with Laplacian filtering, and the more recent $3.8 \AA$ cryo-EM map (Yan et al., 2015) confirmed the location (with an accuracy of $2.1 \AA$ rmsd (Wang, June 22, 2015). Importantly, not all the low-resolution maps had given the same result (only three out of four had it correct), but it was clear from the ranking of the correlation coefficients that the odd one was incorrect (i.e. there was little contrast between correlations coefficients of the top hit and the next ones down in the ranking) (Van Petegem, 2015). To assist Situs users in managing similar ambiguities, we feel it is therefore important to describe best practices for fitting and validation.

As an inspiration for currently supported validation options we recommend the "Supplementary Information" paper of (Tung et al., 2010). In this earlier work, Van Petegem's lab presented a new model of the ABC domains of RyR1. Since their model was in conflict with earlier models, the Situs fitting strategy was extensively scrutinized in that paper. To simplify the listing of best practices, we assume in the following that the fitted subunits are based on reliable crystallographic or NMR structures.

1. Use of two or more independent cryo-EM maps. Since cryo-EM progresses incrementally towards higher resolution maps, it is often possible to compare fitting results to older, lower resolution maps derived from different sets of images. In (Tung et al., 2010) a $14 \AA$ and a $10 \AA$ map of RyR 1 was used to confirm that the model was robust. This idea could be useful to test the robustness of a fitting method or to validate maps. However, the approach does not directly validate models: Any discrepancies arising in such comparisons may be due to weaknesses in one of the maps (typically the older one), which is unrelated to the accuracy of the model derived from the new map.

2. Statistical confidence analysis. It is possible to estimate the precision of the fitting method using a statistical evaluation of the variability of fits. This can be done with a Fisher Z-transform of the scoring function (Volkmann, 2009): For example, a precision of $4.4 \AA$ was reported for fits within the $99 \%$ confidence level for the maximum peak in the cross correlation (Tung et al., 2010). Unfortunately, although the two terms are often confused, precision is not a reliable indicator of accuracy (Henderson et al., 2012). The precision of detected features correlates with the accuracy in single molecule fits where all density is accounted for (Wriggers and Birmanns, 2001), but in cases where a smaller fragment is fitted to a larger map, spurious peaks can arise in the cross-correlation that are far from the true solution (Chacon and Wriggers, 2002).

3. Docking contrast. The docking contrast used by (Lau and Van Petegem, 2014) and (Tung et al., 2010) is a feature of the "scoring function landscape" in the Situs colores tool. It measures how the score of the top solution stands out from non-redundant solutions immediately below in the ranking. The success of this criterion depends on how well the atomic fragments match the EM density. The ranking is less reliable if fitted atomic fragments are imperfect to begin with (for example, when 
homology models are used or in induced fit situations). Also, the use of a Laplacian filter (Chacon and Wriggers, 2002) or multi-fragment docking (see Methods) yield a different contrast profile compared to a one-at-a time approach.

4. Use of two or more independent modeling strategies. We encourage our users to validate their fits with alternative software such as EMFit in which detailed atomic contacts are scored in addition to density matching (Rossmann, 2000). Ideally, different software should yield identical results (Tung et al., 2010). Also, it is useful for maps near $10 \AA$ resolution (or below) to experiment with the Laplacian filter that matches the surface contours (Chacon and Wriggers, 2002). Finally, an important approach fitting smaller fragments is to test whether their matching recapitulates the full assembly: "The results indicate the RyR1ABC structure is at the size limit for standard cross-correlation-based docking into the current $9.6 \AA$ cryo-EM map, and that Laplacian filtering is absolutely required for docking of any smaller units" (Tung et al., 2010).

5. Comparison with other biophysical and biochemical knowledge. This is the most reliable validation strategy because it is independent of the data and modeling work flow. For example, (Tung et al., 2010) studied the distribution of disease mutants in their model. They also evaluated surface features and position of labels as well as results from complementary biophysical and biochemical studies. Fortunately, the expertise of the modeler will be readily available during an interpretation of a cryo-EM map, since functional conclusions are drawn from the atomic model as part of the structure determination process.

The above recommended validation strategies are best practices that will minimize (but not eliminate) the risk of modeling error. Anecdotal evidence suggests that criteria that are solely based on the "scoring landscape" such as confidence analysis (precision) and docking contrast are imperfect predictors of accuracy. For example, it is notoriously difficult to predict the global shape of larger homology models, so their fit might produce unambiguous solutions that have high contrast and high precision, yet the fit may be incorrect (Tung et al., 2010). Also, the use of crystallographic validation methods that evaluate the stereochemistry of protein models are limited in terms of predicting the global pose. For example, the influenza B virus receptor-binding site complex of antibody CR8033 exhibits steric clashes with the hemagglutinin (Dreyfus et al., 2012), but the model is most likely correct since both subunits were solved in isolation and induced fit changes in the EM map of the complex can explain the clashes (Dreyfus et al., 2012). We argue therefore that at the present time a meta-analysis of multiple criteria, that takes into account all experimental knowledge, is the most promising strategy in validation.

\section{Medium Resolution: Tracking by Fiducials}

From a numeric geometry perspective, the above one-at-a-time rigid body docking requires very little information, namely the centroid and pose (orientation) of the docked molecular structure. In other words, the 
fitting accuracy is estimated globally for the entire model. A major limitation of this approach is its inability to differentiate between misalignment and internal conformational change. It would be desirable to add flexibility to the model by introducing sampling points that correspond to specific shape features. The displacement of the sampling points would then account for systematic differences between a map and a model, even when both are in (nearly) equivalent conformations. The magnitude of any conformational change can be quantified by the rmsd of the sampling points, and the drift vectors of individual points could provide a sparsely sampled deformation field. One can indeed devise such a model for flexible fitting of models to low-resolution EM maps (Birmanns and Wriggers, 2007). Fig. 1 illustrates the flexible modeling of the myosin motor domain (Wriggers, 2010). In flexible fitting the deviating atomic structure is brought into register with the EM density by forcing the fiducials to coincide.
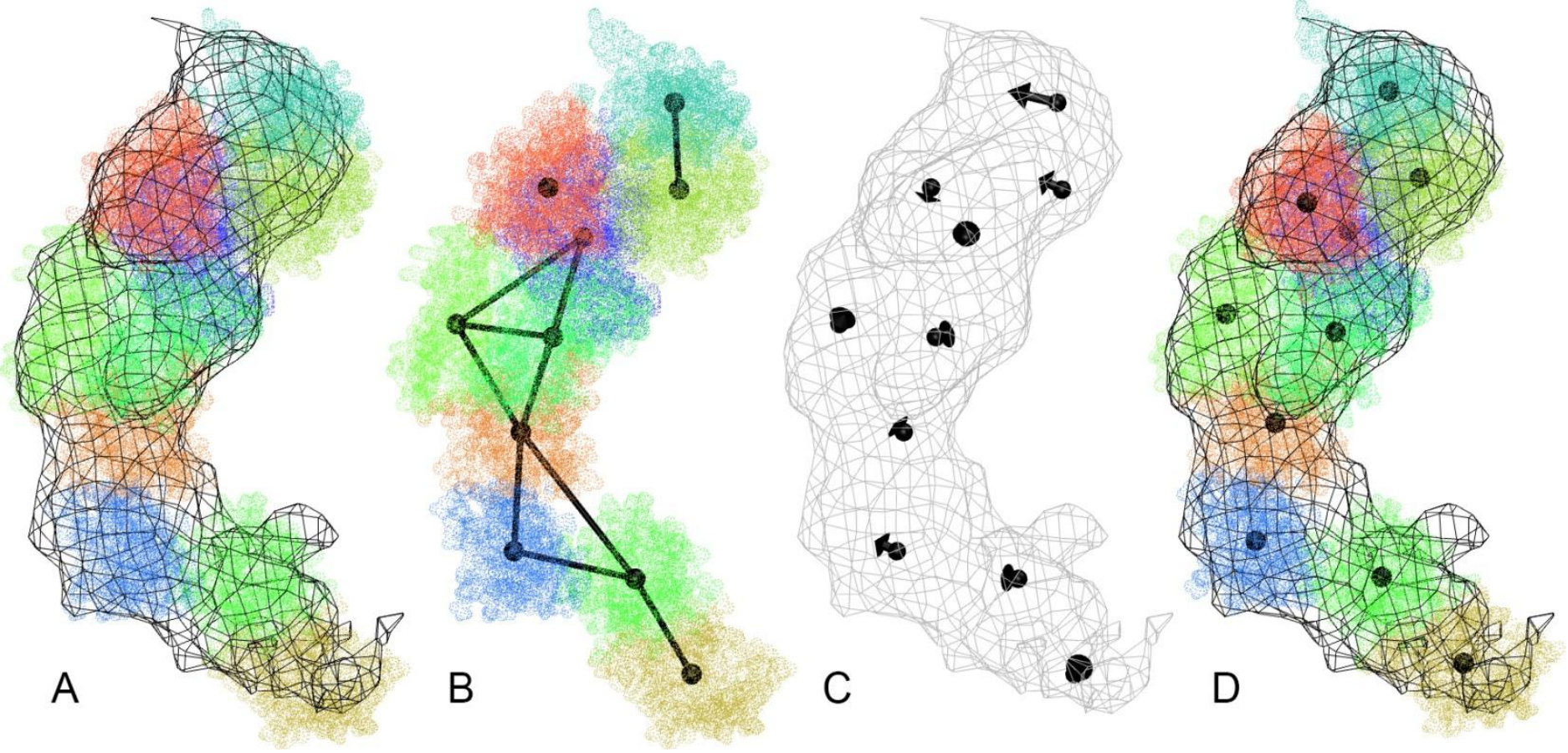

Figure 1: Illustration of the motion capture network (Rusu et al., 2008; Wriggers, 2010), which was originally developed for low-resolution flexing and which serves here as an inspiration for feature tracking. A: crystal structure of the myosin 2 (subfragment 1) motor domain rigid body fitted to the $14 \AA$ resolution EM density (Wriggers, 2010). Atoms are shown as dotted spheres and the EM density is shown as a black wire mesh. The atom colors correspond to Voronoi cells in B. The crystal structure does not match the EM density perfectly as indicated by the imperfect overlap. B: Simulated fiducials (black spheres) used for guiding the flexible fitting of the atomic structure. The colors represent the Voronoi cells (Wriggers et al., 2004) surrounding the fiducials. The fiducials are computed using a so-called vector quantization based on unsupervised learning (Wriggers et al., 2004). The motion capture network (black distance constraints between fiducials) reflects the topology and rigidity of the atomic structure (Wriggers, 2010) and provides a more robust model. C: Discretely sampled displacements as a course representation of the conformational difference. Arrows point from the fiducial position in B to the position in the EM map. D: Flexibly fitted atomic structure superimposed with the EM map. The 
centroids of the Voronoi cells are shifted to their new position. This can be achieved in a MD refinement step or by spatial interpolation (Rusu et al., 2008; Wriggers, 2010). Figures 1 and 2 were created with VMD (Humphrey et al., 1996).

In this work, we are extending the earlier approach to track features in map-model pairs that are deemed of to be equivalent, to search for systematic differences between low to medium resolution EM maps and a known atomic structure. We compare three motion-tracking models. The first model, naïve vector quantization (VQ), involves motion capture, but with the stabilizing skeletons (Fig. 1B) turned off. This is a worst-case scenario, where errors from both model and EM coarse graining can combine. We expect a more robust fit to be provided by direct cross correlation maximization between the EM map and a smoothly deformable model using inverse distance weighting (IDW) interpolation that we developed earlier for flexible fitting (Rusu et al., 2008). Finally, we perform a tessellation of the structure into $\mathrm{N}$ rigid Voronoi cells, which are fitted simultaneously by maximizing the cross correlation in a $6 \mathrm{~N}$-dimensional search (Fig. 2).

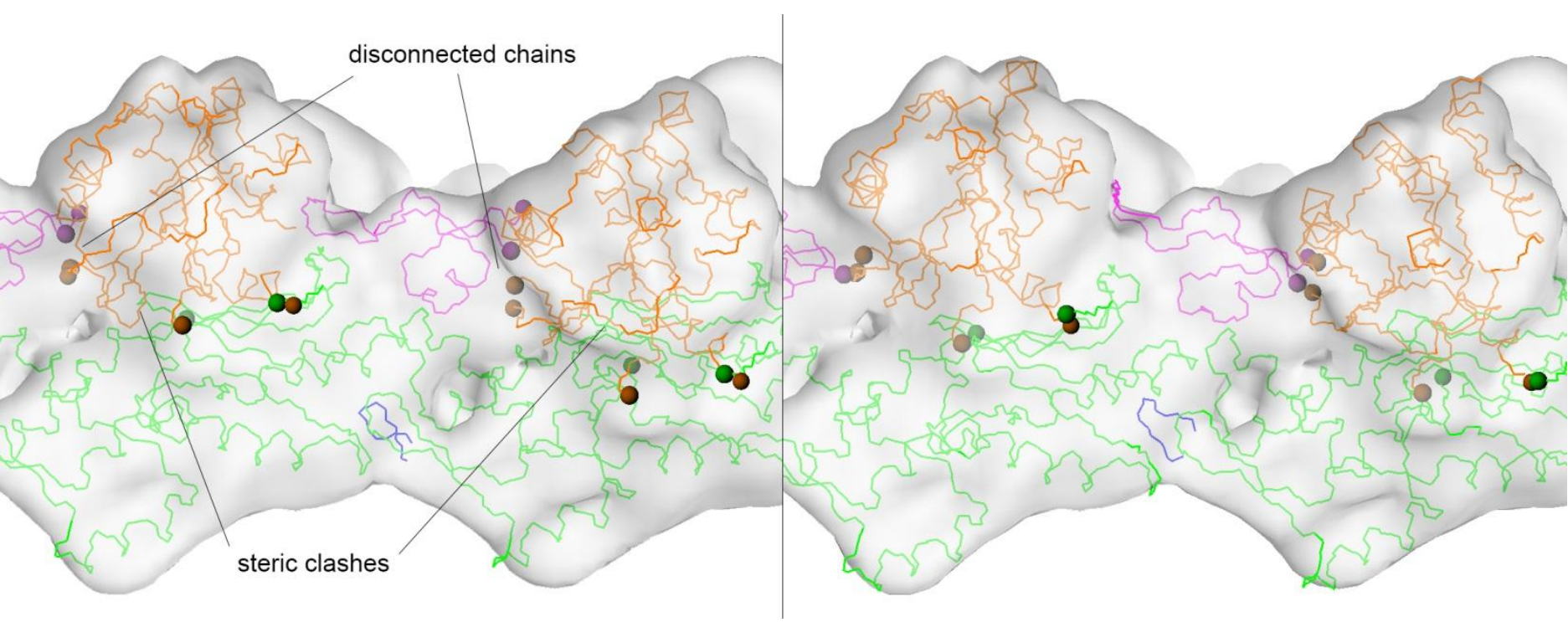

Figure 2: Illustration of the effect of simultaneous multi-fragment fitting. Docking of rigid G-actin subunits (green, purple, orange chains) and phalloidin (blue chain) to an F-actin cryo-EM map at $10.5 \AA$ resolution (data provided by Edward $\mathrm{H}$. Egelman). Shown are two adjacent G-actin symmetry mates within a single strand of the two-start actin helix (-333.8 degrees rotation, 53.2 A translation). Spheres denote ends of the fragmented polypeptide chains within each G-actin. Left: traditional one-at-a-time fitting of the rigid subunits suffers from steric clashes and significant separation in the chain ends. Right: simultaneous multi-fragment fitting provides a cleaner model (with reduced chain separation and no steric clashes) that can be "healed" with local MD simulation that fuses the separated chains. The cross-correlation coefficient optimized here is a widely used fitting score. Its maximization is equivalent to minimizing the squared differences, which is maximum-likelihood if the errors are normal (as is generally assumed in EM fitting applications, although this may not necessarily be the case). This scoring function has been adopted by a large number of groups, and a number of computer programs based on it are readily available (López-Blanco and Chacón, 2015). Over the years, we have developed Fourier-based techniques to accelerate translational or rotational matching (López-Blanco and Chacón, 2015), and the 
colores program has become the workhorse for Situs docking (Wriggers, 2012). (Lasker et al., 2009) originally discovered that the accuracy of traditional interactive component placement, one at a time, can be increased by simultaneous refinement. In the Situs collage tool, the approach softly enforces shape complementarities between components via normalization of the cross correlation (Birmanns et al., 2011). The approach also led to an implementation of symmetry constraints during the refinement (Wriggers, 2012), and it opened the door to a new high-dimensional conformational search (López-Blanco and Chacón, 2013) of multiple fragments, which were optimized in parallel using genetic algorithms in combination with tabu search techniques (Rusu and Birmanns, 2010). We have recently adopted this approach, termed voltrac, to trace $\alpha$-helices in high-resolution EM maps (Rusu and Wriggers, 2012) and to segment filaments in tomograms (Rusu et al., 2012).

We compare in Fig. 3 the structure of GroEL (PDB ID 1XCK) with an $11.5 \AA$ resolution map (EMDB ID 1080) and with a $6 \AA$ resolution map (EMDB ID 1081). The three structures are all for the wild-type specimen and are expected to be equivalent. Our stability tests should yield very small deviations for the EM features from those corresponding to the known crystal structure. We carried out calculations for the more efficient VQ approach up to $\mathrm{N}=500$, which is just below the maximum meaningful number $\mathrm{N}_{\max }$ of features contained in EMDB ID 1080 (this number can be estimated from the occupied volume divided by the volume of a cubic resolution element and yields $N_{\max } \sim 660$ for the $11.5 \AA$ map and $N_{\max } \sim 4600$ for the $6 \AA$ map). Calculations for the computationally more demanding global deformation and Voronoi tessellation models were carried out up to $\mathrm{N}=100$

The observed rmsd values give insight into discrepancies between the structures and allow us to quantify the effect of imperfect docking strategies. All three approaches initially increase their rmsd as the models become more flexible due to increasing number of features. The VQ approach requires sufficient point density $(\mathrm{N}>100)$ to track features consistently. VQ is somewhat complementary to the other two approaches (due to its ability to easily reach high $\mathrm{N}$ on standard workstations), but it suffers from intermittent bursts of instability at lower $\mathrm{N}$ and even at high $\mathrm{N}$ the curves are not as smooth as those computed by the other approaches. A more consistently accurate fit at lower $\mathrm{N}$ is provided by the deformable model using IDW interpolation (Rusu et al., 2008). The observed rmsd varies smoothly between 1 and $2 \AA$. Finally, the lowest rmsd is consistently provided by tessellation of the structure into rigid Voronoi cells utilizing multi-fragment refinement (Fig. 2). This lowest error is an upper bound for the (small) discrepancy between crystal structure and EM map due to different experimental conditions. Given that the best performing Voronoi tessellation approach produces a low rmsd below $2 \AA$, and the $V Q$ approach for high $N$ is saturating in both cases, we believe that the maximum Voronoi rmsd is close to the (unknown) structure discrepancy. Interestingly, there is little difference between the Voronoi plots of the $11.5 \AA$ and the $6 \AA$ maps, which also supports the interpretation that the discrepancy is due to a systematic difference between crystal structure and both EM reconstructions. Therefore, we have found a case where the results would argue against over-fitting the atomic structure (e.g. by using an aggressive molecular dynamics approach that would erase all meaningful differences between map and model). 
Figure 3: Accuracy of tracking by fiducials. PDB ID 1XCK vs. EMDB ID 1080 (top; GroEL at $11.5 \AA$ resolution) and EMDB ID 1081 (bottom; GroEL at $6 \AA$ resolution). Shown are feature drift rmsd values observed by (1) naïve vector quantization [(Wriggers et al., 2000), black dots], (2) a global deformable model based on IDW interpolation [(Rusu et al., 2008), red], and (3) rigid-body multi-fragment model based on Voronoi tessellation (green).

\section{Medium to High Resolution: Validation using Secondary Structure Elements?}

Given the increasing number of available cryo-EM density maps in the medium to high resolution range, secondary structure features of cryo-EM maps have been well characterized and their features are now well understood. In fact, secondary structure elements are often the most obvious features in density maps at medium to high resolution. Consequently, we argue in this section that it is useful to generalize the above point-wise tracking (Fig. 3) to more abstract representations of geometric shape that are tuned to the specific properties of secondary structure elements. In general, long helices can be detected reliably and $\beta$-sheets also begin to become visible at resolutions of $8 \AA$ or better (Baker et al., 2012). It is also possible to predict the location of $\beta$-strands at medium resolution ( $\mathrm{Si}$ and $\mathrm{He}, 2014 \mathrm{a}$ ).

Secondary structures have unique density signatures. Helices, particularly long helices appear as cylinders, and simple sheets appear as thin twisted layers of density (Fig. 4). Since Jiang et al. discovered that it is 
possible to identify helices in sub-nanometer resolution maps (Jiang et al., 2001), various computational methods have been developed to detect helices and $\beta$-sheets, including SSEhunter, SSETracer and voltrac (Baker et al., 2007; Dal Palu et al., 2006; Kong and Ma, 2003; Kong et al., 2004; Rusu and Wriggers, 2012; Si and He, 2013 ; Si et al., 2012; Zeyun and Bajaj, 2008). SSELearner combines image processing techniques and machine learning ( $\mathrm{Si}$ et al., 2012). An example of the helices (red sticks) and $\beta$-sheets (green volume) detected using SSETracer is shown in Figure 4B. It is also possible to obtain a skeleton (pink) that represents possible connected components in the density map (Figure 4B). Recently, StrandTwister was developed to detect $\beta$-strands (red lines in Figure 4C) from $\beta$-sheet density through the analysis of $\beta$-sheet twist (Si and $\mathrm{He}$, 2014a). The results generated from such detection methods reflect the strength of the density features at secondary structure regions.
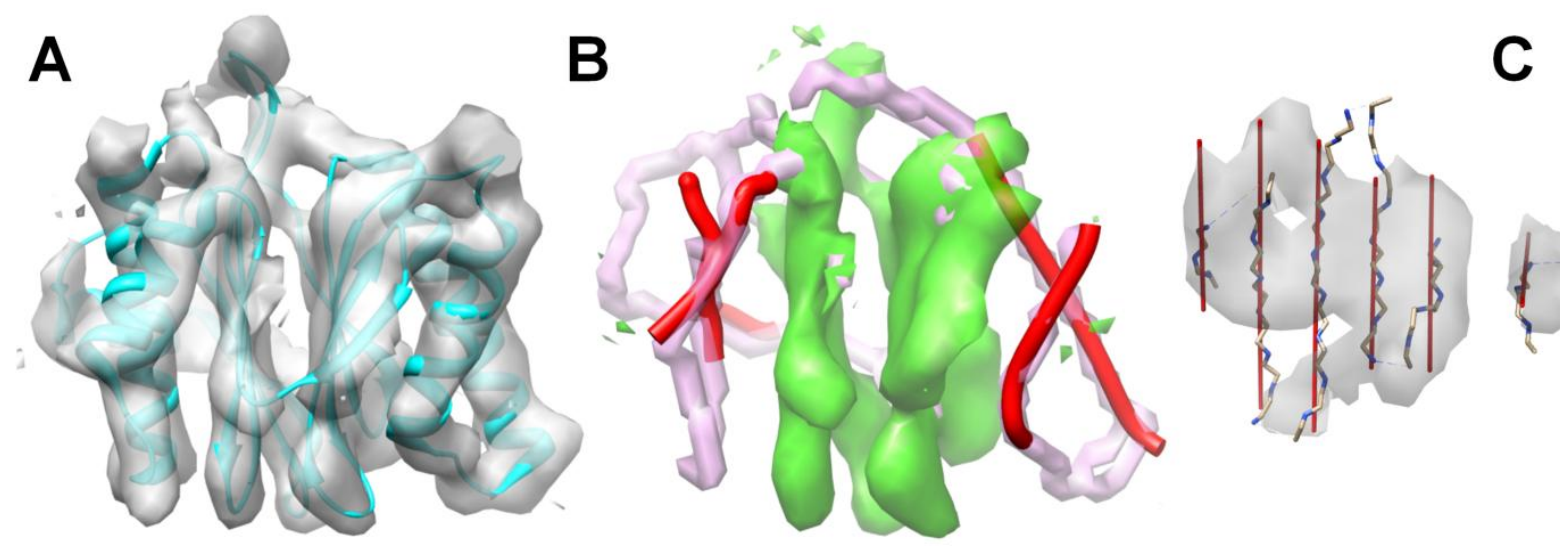

Figure 4: Secondary structure detection based on characteristic density patterns. A: The density map (gray) extracted from EMDB 1733 (6.8 ̊ resolution) superimposed on its atomic structure (cyan; PDB 3FIN). B: The detected helices (represented as red sticks) and two $\beta$-sheets (green density), using SSETracer (Si and He, 2013) and the skeleton (pink) using SkelEM (AI Nasr et al., 2013). C: The best of the top ten sets of detected $\beta$-traces (red lines) using StrandTwister (Si and $\mathrm{He}, 2014 \mathrm{a}$ ) are superimposed with the backbone of the $\beta$-strands and the density maps (gray) for EMDB ID 2165 and PDB ID 4B4T. Figures 4 and 5 were created using Chimera (Pettersen et al., 2004).

Unfortunately, due to the higher resolution in today's cryo-EM maps, it is quite common to see quality variation in the density of secondary structure elements (Fig. 5). For example, the upper left helix in Figure 5 exhibits a strong cylinder-like shape, while the upper middle helix shows thinner and discontinuous pieces of a cylinder in the same density map at the same threshold. These quality variations might be due to uneven resolution across the map, 3D reconstruction artifacts, or heterogeneity of the conformation. Likewise, it is possible that a matched atomic model does not accurately reflect the EM density due to limitations of the modeling or fitting. Given the increasing number of map-model pairs deposited in the EMDB and PDB, we argue that there is a need to quantify the fitting locally to distinguish those models that agree well with the local density features from those that do not. We have provided Figure 5 as an illustration of such cases which should be useful to modelers in the community who develop such validation software. 


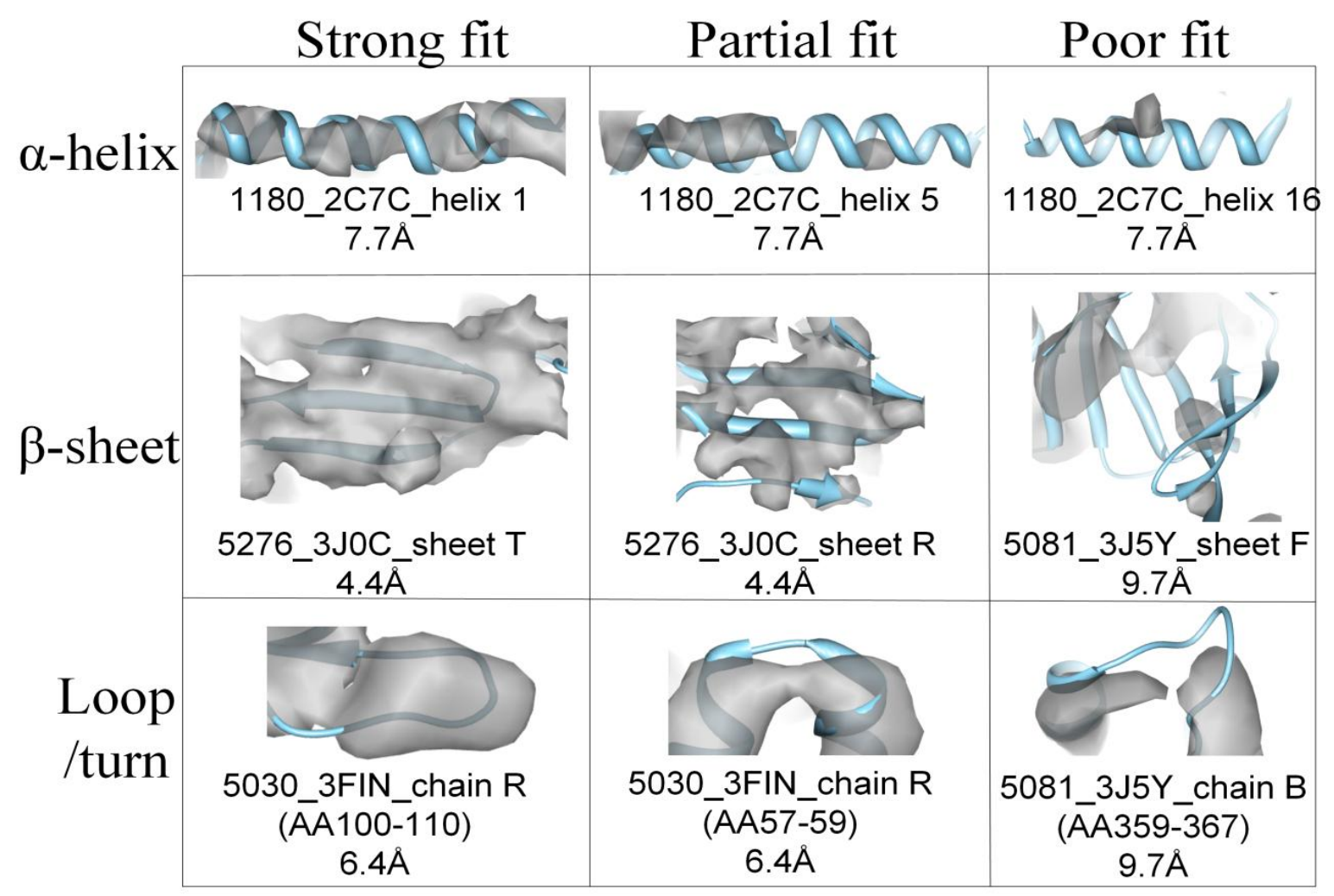

Figure 5: Quality variation in density maps at secondary structure regions. Each local region is labeled with EMDB ID, PDB ID, and resolution of the cryo-EM map. The atomic model (blue ribbon) is aligned with a local density pattern (gray) in the pose taken from the database.

Secondary feature representations can be used to define metrics for evaluating the local fitness of a mapmodel pair. For example, the helical axes and beta strands in Figure 4B and $C$ could be used to measure lateral helical or strand displacement, or any longitudinal length discrepancy. The density property of secondary structure elements is already widely used for the validation of map-model pairs. Helical density has been characterized using various metrics such as cross correlation coefficients with cylindrical templates (Baker et al., 2007; Rusu and Wriggers, 2012), gradient tree (Dal Palu et al., 2006) and local structural tensor(Si and He, 2013; Si et al., 2012; Zeyun and Bajaj, 2008). These methods characterize the cylindrical property along the axis of the helix. The continuity of high density along the axis and the local thickness of the cylindrical density are often indicators of the strength of the helix density feature. The density feature of a $\beta$ sheet is less well explored compared to that of a helix. Nevertheless, thinning and pruning has been shown to derive the surface of a $\beta$-sheet (Baker et al., 2007). Recent studies suggested that the twist of a $\beta$-sheet can also be exploited, even if the strands themselves cannot be visualized (Si and He, 2014a; Si and He, 2014b).

In summary, we are witnessing the development of quantitative secondary structure-based methods that are able to characterize local discrepancies in a map-model pair. This area of research is in its infancy now but we expect that it will grow much more important in the near future. 


\section{Conclusions} multiple subunits.

\section{Availability}

Our tests suggests that multi-fragment rigid body refinement is a superior "motion" model for tracking deformations, and that Voronoi cells of fiducials can be used as templates for tracking conformational differences. This is another successful adaptation of the new fitting paradigm of simultaneous refinement of

Our work also showed that flexible fitting methods such as all-atom molecular dynamics may accidentally erase meaningful differences between atomic structures and EM maps if they reduce the discrepancy between models and maps to zero. This is a problem that mostly affects medium to low resolution maps where the fitted atomic structure might be more reliable than the map density. The ultimate remedy to this problem is higher resolution that allows cryo-EM to solve atomic structures directly. Nevertheless, medium to low resolution maps are still dominating the EMDB. It is therefore important to separate flexibly fitted models from rigid ones in a statistical evaluation of map-model pairs.

We also presented various map-model pairs that are potentially useful for testing validation methods based on secondary structure elements. Our "call to arms" seeks to shift the research emphasis from qualitative detection of secondary structures to quantitative validation of secondary structure patterns. This approach would open the door to new applications in the validation of atomic structures based on secondary structure patterns, it would enrich existing detection methods for better reliability, and it would provide a local measure of fitness for maps in case of reliable reference structures.

Tools and tutorials for the numerical experiments in this paper will be made available with version 2.8 of Situs (http://situs.biomachina.org).

\section{Acknowledgements}


We thank Catherine Lawson, Hongwei Wang and Filip Van Petegem for discussions and Edward H. Egelman for providing cryo-EM data. We thank Dong Si, Lin Chen for preparing figures. This work is supported by $\mathrm{NIH}$ R01 GM062968 and by NSF DBI-1356621.

\section{References}

Al Nasr, K., Liu, C., Rwebangira, M., Burge, L., He, J., 2013. Intensity-based skeletonization of CryoEM gray-scale images using a true segmentation-free algorithm. IEEE/ACM transactions on computational biology and bioinformatics / IEEE, ACM 10, 1289-1298.

Baker, M.L., Ju, T., Chiu, W., 2007. Identification of secondary structure elements in intermediate-resolution density maps. Structure 15, 7-19.

Baker, M.L., Baker, M.R., Hryc, C.F., Ju, T., Chiu, W., 2012. Gorgon and pathwalking: macromolecular modeling tools for subnanometer resolution density maps. Biopolymers 97, 655-668.

Birmanns, S., Wriggers, W., 2007. Multi-resolution anchor-point registration of biomolecular assemblies and their components. Journal of structural biology 157, 271-280.

Birmanns, S., Rusu, M., Wriggers, W., 2011. Using Sculptor and Situs for simultaneous assembly of atomic components into low-resolution shapes. Journal of structural biology 173, 428-435.

Chacon, P., Wriggers, W., 2002. Multi-resolution contour-based fitting of macromolecular structures. Journal of molecular biology 317, 375-384.

Dal Palu, A., He, J., Pontelli, E., Lu, Y., 2006. Identification of Alpha-Helices from Low Resolution Protein Density Maps. Proceeding of Computational Systems Bioinformatics Conference(CSB), 89-98.

DiMaio, F., Tyka, M.D., Baker, M.L., Chiu, W., Baker, D., 2009. Refinement of protein structures into low-resolution density maps using rosetta. Journal of molecular biology 392, 181-190.

Dreyfus, C., Laursen, N.S., Kwaks, T., Zuijdgeest, D., Khayat, R., Ekiert, D.C., Lee, J.H., Metlagel, Z., Bujny, M.V., Jongeneelen, M., van der Vlugt, R., Lamrani, M., Korse, H.J., Geelen, E., Sahin, O., Sieuwerts, M., Brakenhoff, J.P., Vogels, R., Li, O.T., Poon, L.L., Peiris, M., Koudstaal, W., Ward, A.B., Wilson, I.A., Goudsmit, J., Friesen, R.H., 2012. Highly conserved protective epitopes on influenza B viruses. Science 337, 1343-1348.

Emsley, P., Lohkamp, B., Scott, W.G., Cowtan, K., 2010. Features and development of Coot. Acta crystallographica. Section D, Biological crystallography 66, 486-501.

Henderson, R., Sali, A., Baker, M.L., Carragher, B., Devkota, B., Downing, K.H., Egelman, E.H., Feng, Z., Frank, J., Grigorieff, N., Jiang, W., Ludtke, S.J., Medalia, O., Penczek, P.A., Rosenthal, P.B., Rossmann, M.G., Schmid, M.F., Schroder, G.F., Steven, A.C., Stokes, D.L., Westbrook, J.D., Wriggers, W., Yang, H., Young, J., Berman, H.M., Chiu, W., Kleywegt, G.J., Lawson, C.L., 2012. Outcome of the first electron microscopy validation task force meeting. Structure 20, 205-214.

Humphrey, W., Dalke, A., Schulten, K., 1996. VMD: visual molecular dynamics. Journal of molecular graphics 14, 33-38, 27-38.

Jiang, W., Baker, M.L., Ludtke, S.J., Chiu, W., 2001. Bridging the information gap: computational tools for intermediate resolution structure interpretation. J Mol Biol 308, 1033-1044.

Jin, L., Milazzo, A.C., Kleinfelder, S., Li, S., Leblanc, P., Duttweiler, F., Bouwer, J.C., Peltier, S.T., Ellisman, M.H., Xuong, N.H., 2008. Applications of direct detection device in transmission electron microscopy. Journal of structural biology 161, 352-358.

Kong, Y., Ma, J., 2003. A structural-informatics approach for mining beta-sheets: locating sheets in intermediateresolution density maps. J Mol Biol 332, 399-413.

Kong, Y., Zhang, X., Baker, T.S., Ma, J., 2004. A Structural-informatics approach for tracing beta-sheets: building pseudoC(alpha) traces for beta-strands in intermediate-resolution density maps. J Mol Biol 339, 117-130.

Lasker, K., Topf, M., Sali, A., Wolfson, H.J., 2009. Inferential Optimization for Simultaneous Fitting of Multiple Components into a CryoEM Map of Their Assembly. Journal of Molecular Biology 388, 180-194.

Lau, K., Van Petegem, F., 2014. Crystal structures of wild type and disease mutant forms of the ryanodine receptor SPRY2 domain. Nature communications 5, 5397. 
1 Lawson, C.L. 2014. Pers. Comm.

López-Blanco, J.R., Chacón, P., 2013. iMODFIT: efficient and robust flexible fitting based on vibrational analysis in internal coordinates. Journal of structural biology 184, 261-270.

López-Blanco, J.R., Chacón, P., 2015. Structural modeling from electron microscopy data. Wiley Interdisciplinary Reviews: Computational Molecular Science 5, 62-81.

Pettersen, E.F., Goddard, T.D., Huang, C.C., Couch, G.S., Greenblatt, D.M., Meng, E.C., Ferrin, T.E., 2004. UCSF ChimeraA visualization system for exploratory research and analysis. Journal of Computational Chemistry 25, 1605-1612.

Rossmann, M.G., 2000. Fitting atomic models into electron-microscopy maps. Acta crystallographica. Section D, Biological crystallography 56, 1341-1349.

Rossmann, M.G., Bernal, R., Pletnev, S.V., 2001. Combining electron microscopic with x-ray crystallographic structures. Journal of structural biology 136, 190-200.

Rusu, M., Birmanns, S., 2010. Evolutionary tabu search strategies for the simultaneous registration of multiple atomic structures in cryo-EM reconstructions. Journal of structural biology 170, 164-171.

Rusu, M., Wriggers, W., 2012. Evolutionary bidirectional expansion for the tracing of alpha helices in cryo-electron microscopy reconstructions. Journal of structural biology 177, 410-419.

Rusu, M., Birmanns, S., Wriggers, W., 2008. Biomolecular pleiomorphism probed by spatial interpolation of coarse models. Bioinformatics 24, 2460-2466.

Rusu, M., Starosolski, Z., Wahle, M., Rigort, A., Wriggers, W., 2012. Automated tracing of filaments in 3D electron tomography reconstructions using Sculptor and Situs. Journal of structural biology 178, 121-128.

Si, D., He, J., 2013. Beta-sheet Detection and Representation from Medium Resolution Cryo-EM Density Maps. BCB'13: Proceedings of ACM Conference on Bioinformatics, Computational Biology and Biomedical Informatics Washington, D.C., 764-770.

Si, D., He, J., 2014a. Tracing beta-strands using strandtwister from cryo-EM density maps at medium resolutions. Structure 22, 22(11):1665-1676.

Si, D., He, J. 2014b. Orientations of beta-strand traces and near maximum twist, pp. 690-694 Proceedings of the 5th ACM Conference on Bioinformatics, Computational Biology, and Health Informatics. ACM, Newport Beach, California.

$\mathrm{Si}, \mathrm{D} ., \mathrm{Ji}, \mathrm{S} ., \mathrm{Nasr}, \mathrm{K} . \mathrm{A} ., \mathrm{He}$, J., 2012. A machine learning approach for the identification of protein secondary structure elements from electron cryo-microscopy density maps. Biopolymers 97, 698-708.

Trabuco, L.G., Villa, E., Mitra, K., Frank, J., Schulten, K., 2008. Flexible fitting of atomic structures into electron microscopy maps using molecular dynamics. Structure 16, 673-683.

Tung, C.C., Lobo, P.A., Kimlicka, L., Van Petegem, F., 2010. The amino-terminal disease hotspot of ryanodine receptors forms a cytoplasmic vestibule. Nature $468,585-588$.

Van Petegem, F., 2015. Pers. Comm.

Volkmann, N., 2009. Confidence intervals for fitting of atomic models into low-resolution densities. Acta crystallographica. Section D, Biological crystallography 65, 679-689.

Wang, H. June 22, 2015. Pers. Comm.

Wriggers, W., 2010. Using Situs for the integration of multi-resolution structures. Biophysical reviews 2, 21-27.

Wriggers, W., 2012. Conventions and workflows for using Situs. Acta crystallographica. Section D, Biological crystallography $68,344-351$.

Wriggers, W., Birmanns, S., 2001. Using situs for flexible and rigid-body fitting of multiresolution single-molecule data. Journal of structural biology 133, 193-202.

Wriggers, W., Agrawal, R.K., Drew, D.L., McCammon, A., Frank, J., 2000. Domain motions of EF-G bound to the $70 \mathrm{~S}$ ribosome: insights from a hand-shaking between multi-resolution structures. Biophysical journal 79, 1670-1678.

Wriggers, W., Chacon, P., Kovacs, J.A., Tama, F., Birmanns, S., 2004. Topology representing neural networks reconcile biomolecular shape, structure, and dynamics. Neurocomputing 56, 365-379.

Yan, Z., Bai, X.C., Yan, C., Wu, J., Li, Z., Xie, T., Peng, W., Yin, C.C., Li, X., Scheres, S.H., Shi, Y., Yan, N., 2015. Structure of the rabbit ryanodine receptor RyR1 at near-atomic resolution. Nature 517, 50-55.

Zeyun, Y., Bajaj, C., 2008. Computational Approaches for Automatic Structural Analysis of Large Biomolecular Complexes. Computational Biology and Bioinformatics, IEEE/ACM Transactions on 5, 568-582. 